\title{
RETOS DE FUTURO DE LA PSICOLOGÍA CLÍNICA
}

\author{
Enrique Echeburúa \\ Departamento de Personalidad, \\ Evaluación y Tratamiento Psicológico \\ Facultad de Psicología, Universidad del País Vasco
}

\section{RESUMEN}

Los retos futuros de la psicología clínica son analizados en este artículo. Los pacientes buscan actualmente ayuda terapéutica no sólo por los trastornos mentales tradicionales. La psicología clínica basada en la evidencia implica la integración de la experiencia clínica con los resultados de la investigación. Sin embargo, hay muchas discrepancias entre la investigación y la práctica clínica: no siempre un tratamiento eficaz en un ensayo clínico resulta efectivo en la práctica clínica habitual. A pesar de los grandes progresos en la validación de los tratamientos eficaces, su difusión a los profesionales clínicos es aún muy limitada. Se analizan los últimos desarrollos para conseguir una mayor colaboración entre los investigadores y los clínicos. Se abordan asimismo cuestiones éticas relevantes en relación con la práctica clínica y se analizan las características psicológicas del terapeuta que están más relacionadas con el éxito terapéutico.

Palabras clave: Psicología clínica. Terapias validadas empíricamente. Difusión de las terapias efectivas. Cuestiones éticas en la práctica clínica. 


\section{SUMMARY}

Future challenges for clinical psychology are reviewed in this paper. Patients are now seeking treatment not only for traditional mental disorders. Evidence-based psychological practice involves integrating clinical expertise with the best available external evidence from systematic research. However, there are lot of discrepancies between research and practice: not always an efficacious treatment can be transported from the clinic research to community and private settings. Dissemination of evidence-based therapies to mental health professionals is still limited. Recent developments to foster a more productive collaboration between clinicians and researchers are considered. Relevant ethical questions about clinical practice, as well as psychological features of therapists, are also discussed.

\section{Key words: Clinical psychology. Evidence-based psy- chological practice. Dissemination of effective therapies. Ethical questions in psychological practice.}

\section{INTRODUCCIÓN}

Lo que debe saber un psicólogo clínico -y también lo que puede y debe hacer- es objeto continuo de controversia. Asimismo el papel profesional del psicólogo, muy en consonancia, por otra parte, con la formación recibida, es un tema debatido permanentemente. No hay más que echar un vistazo, por ejemplo, a algunos libros sugerentes (Avia, 2000) o a la trayectoria de la revista Papeles del Psicólogo, editada por el Consejo General de Colegios Oficiales de Psicólogos de España, para percatarse de esta realidad (Echeburúa, Corral y Salaberría, 2005).

La psicología clínica ha experimentado un cambio espectacular en los últimos 25 años. En concreto, se ha evolucionado de un ámbito basado fundamentalmente en el psicodiagnóstico, con una aplicación muy limitada al campo de la terapia, a un ámbito mucho más integrado, que abarca la evaluación y los tratamientos psicológicos, el diseño de programas de investigación clínica o la elaboración de 
estudios epidemiológicos. En último término, la psicología clínica es una disciplina que en la actualidad describe los trastornos psicológicos, los evalúa adecuadamente, genera unas técnicas de intervención y se articula en una red asistencial determinada.

No deja de ser sorprendente que haya inventariadas más de 250 terapias psicológicas diferentes. Que en EE. UU. y en Europa florezcan actualmente una plétora de centros de crecimiento personal, grupos gestálticos, seminarios de percepción extrasensorial, etcétera, no es, como resulta fácil imaginarlo, una cuestión de creatividad científica. Esta variabilidad en la práctica clínica no está relacionada con la evidencia científica o con el juicio clínico. Por ello, no es razonable mantener, especialmente en los centros públicos, terapias que no han mostrado ser eficaces más allá de un efecto placebo o de los efectos inespecíficos de cualquier terapia (Bayés, 1984).

El objetivo de este artículo es plantear una reflexión personal sobre la psicología clínica como disciplina científica, sobre la formación del psicólogo clínico y sobre su conexión con las necesidades profesionales y con las expectativas de la sociedad en el siglo XXI. De este modo, se aborda el tema de las terapias empíricamente validadas y de las nuevas tendencias, pero también se señalan sus limitaciones. $Y$ también se hace referencia a algunos temas menos de moda (pero, sin embargo, extraordinariamente relevantes), como son los aspectos deontológicos implicados en la práctica profesional o el análisis de los requisitos psicológicos necesarios (o, al menos, convenientes) para el ejercicio profesional de la psicología clínica.

Estos puntos de vista constituyen, como no puede ser de otra manera, un planteamiento abierto surgido al hilo de los temas que me parecen más relevantes en el quehacer cotidiano del psicólogo clínico. Al tratarse de unas reflexiones personales, se ha evitado en la medida de lo posible el academicismo al uso y, por ello, se ha restringido el número de las referencias bibliográficas.

\section{NUEVAS DEMANDAS TERAPÉUTICAS}

Las demandas terapéuticas a los psicólogos clínicos y a los psiquiatras han cambiado considerablemente en los últimos años. Ahora se tiende a consultar, además de por los cuadros clínicos tradicio- 
nales (depresión, trastornos de ansiedad, esquizofrenia, adicciones, etcétera), por problemas menores, derivados de una exigencia de una mayor calidad de vida por parte de los pacientes. Estos problemas, que no constituyen propiamente trastornos mentales, reflejan una patología del sufrimiento o de la infelicidad. Entre ellos se encuentran el duelo por la pérdida de un ser querido, los conflictos de pareja o la ruptura de pareja no deseada, las dificultades de convivencia con hijos adolescentes, los problemas de estrés laboral, el dolor crónico, etcétera.

En las clasificaciones psiquiátricas estos problemas adaptativos se denominan códigos $Z$ y son un reflejo de la psicopatologización de las dificultades de la vida cotidiana, así como de los efectos de la soledad y de la incomunicación. Y el volumen de las consultas de este tipo puede suponer casi el $20 \%$ de las demandas asistenciales en un Centro de Salud Mental. Así, por ejemplo, en algunos Centros de Salud Mental el número de consultas derivadas del estrés laboral es igual a la suma de las consultas relacionadas con los trastornos de la conducta alimentaria y con la dependencia del alcohol.

Estas nuevas demandas terapéuticas están relacionadas con una mayor exigencia de calidad de vida por parte de los pacientes y con una mayor intolerancia al malestar emocional, pero también con la medicalización de la vida cotidiana. De hecho, hay una tendencia malsana por asignar a todos los problemas un nombre clínico, unos síntomas y un tratamiento: es una tendencia que se ve acentuada por el marketing de la industria farmacéutica dirigido a médicos y pacientes. No deja de ser significativo, por ejemplo, que la primera edición del DSM (DSM-I, 1952) contenía unos 60 trastornos mentales y que la actual (DSM-IV-TR, 2000) recoja más de 300 . No parece razonable pensar que en menos de 50 años los trastornos mentales se hayan multiplicado por cinco. Por ello, hay que estar precavido ante los supuestos nuevos diagnósticos clínicos: síndrome post-vacacional, síndrome de Diógenes, vigorexia, síndrome de alienación parental, síndrome de Ulises, etcétera.

En resumen, muchas de las consultas a los psicólogos clínicos hoy no se relacionan con trastornos mentales, sino con situaciones de infelicidad. Se trata de personas que se sienten sobrepasadas en sus estrategias de afrontamiento para las dificultades cotidianas y 
que, muy frecuentemente, carecen de una red de apoyo familiar y social sólida. Los psicólogos clínicos tienen que adaptarse a esta nueva realidad, evitar la tendencia a establecer diagnósticos psiquiátricos y desarrollar unas estrategias de intervención (técnicas de counselling, intervención en crisis, etcétera) que no son exactamente las mismas que han mostrado éxito en el tratamiento de los trastornos mentales propiamente dichos. Éste es un reto de futuro importante.

\section{TERAPIAS PSICOLÓGICAS BASADAS EN LA EVIDENCIA}

En los últimos 20 años se han producido tres avances muy importantes en el campo de los trastornos mentales. En primer lugar, se han hecho grandes adelantos en la comprensión de las bases biológicas de muchos cuadros clínicos (la esquizofrenia, la depresión o los trastornos bipolares, por ejemplo). En segundo lugar, se ha desarrollado una nueva generación de psicofármacos (antidepresivos y neurolépticos especialmente), con una mayor eficacia terapéutica y un perfil más favorable de efectos secundarios. $Y$ en tercer lugar -pero no menos importante-, se han creado tratamientos psicológicos breves y efectivos, fundamentalmente de tipo cognitivo-conductual, para una amplia variedad de trastornos (Bados, García y Fusté, 2002).

Sin embargo, la difusión de estos tres avances no ha ido a la par. En los dos primeros casos las compañías farmacéuticas multinacionales se han ocupado de difundirlos ampliamente mediante diversas publicaciones gratuitas (folletos, revistas, libros, etcétera) y congresos financiados. Por el contrario, en el tercer caso -el desarrollo de los tratamientos psicológicos-, al no contar con un órgano de difusión tan poderoso como la industria farmacéutica (que, por motivos obvios, no está interesada en este tema), los avances espectaculares habidos se han limitado a las revistas científicas y no han llegado siquiera a los sectores profesionales implicados ni se les ha prestado la atención debida (Echeburúa y Corral, 2001).

De hecho, los tratamientos psicofarmacológicos (especialmente en el caso de los antidepresivos y de los neurolépticos) prescritos en los centros clínicos han cambiado considerablemente en los últimos años al hilo de los nuevos descubrimientos, pero, sin embargo, los 
avances en las terapias psicológicas no se han reflejado en la práctica clínica habitual ni siquiera a veces en los contenidos formativos de los programas de psicología clínica.

\section{Ventajas}

La valoración de la eficacia de las intervenciones terapéuticas es una necesidad perentoria. En primer lugar, porque el progreso científico de la psicología clínica requiere la delimitación de las terapias eficaces, así como de los componentes activos de las mismas. En segundo lugar, porque esta disciplina se enfrenta, como fruto de su propio desarrollo, a retos de cada vez mayor complejidad (por ejemplo, al tratamiento de los trastornos de personalidad, de los problemas de la conducta alimentaria o de las nuevas adicciones). Y en tercer lugar -pero no menos importante-, porque las terapias psicológicas están ya incorporadas a los Centros de Salud Mental públicos. De este modo, la Sanidad Pública sólo debe ofertar terapias eficaces $y$, en igualdad de condiciones, breves. Es decir, acortar el sufrimiento del paciente y ahorrar gastos y tiempo a los Centros parecen objetivos prioritarios (Echeburúa, 1998).

Las ventajas de la psicología clínica basada en la evidencia son claras. Los organismos públicos y las compañías de seguros pueden contar con criterios operativos para financiar selectivamente sólo las terapias psicológicas empíricamente validadas. A su vez, los pacientes pueden contar con una información clara para saber qué tipo de ayuda buscar para hacer frente a sus problemas psicológicos. Y, finalmente, desde una perspectiva deontológica o judicial, se puede definir operativamente el concepto de mala praxis (cuando el terapeuta se aparta de una práctica profesional empíricamente validada para un trastorno concreto y en unas circunstancias determinadas).

La psicología clínica basada en la evidencia ha contribuido también a delimitar la duración de los tratamientos efectivos. En general, con una terapia psicológica entre 8 y 24 sesiones se obtiene, según los cuadros clínicos de que se trate, una mejoría del $70 \%$ (Turner, Beidel, Spaulding y Brown, 1995). Prolongar la terapia más allá de 24 sesiones y alargarla a 12 meses aumenta muy poco el porcentaje de mejoría e incrementa enormemente el gasto (figura 1). Huelga decir que las psi- 
coterapias psicoanalíticas, que cuentan con 600 a 1000 sesiones por término medio, quedan fuera por completo de este contexto.

\section{FIGURA 1 \\ RELACIÓN Nº DE SESIONES-EFECTOS \\ TERAPÉUTICOS (Howard et al., 1986)}

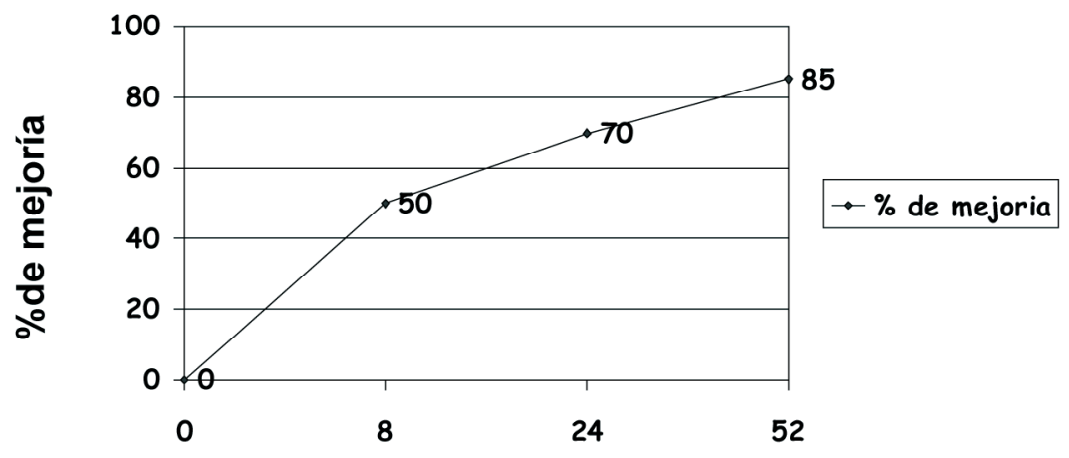

Número de sesiones

\section{Limitaciones}

Las terapias empíricamente validadas han concedido una gran importancia a la clasificación de los trastornos mentales. Esta preocupación por el enfoque científico ha llevado a sobrevalorar el objeto de la psicología (los cuadros clínicos) y a desatender, al menos hasta cierto punto, al sujeto de la psicología (el ser humano pensante, sintiente o, según los casos, sufriente). Es decir, la importancia concedida al diagnóstico clínico (por ejemplo, tratamientos efectivos para el trastorno obsesivo-compulsivo) ha diluido la atención específica al paciente que padece esa patología y no ha tomado en consideración las diferencias individuales existentes entre los pacientes catalogados con el mismo diagnóstico. En este sentido, hay una tendencia a una cierta cosificación del paciente. De este modo, los manuales o guías de tratamiento pueden, a veces, responder (si no se usan bien) a la mentalidad del libro de recetas de cocina. 
La psicología clínica basada en la evidencia está sujeta a diversas limitaciones: las diferencias de rendimiento de los terapeutas con una misma terapia, la variación de la gravedad de los pacientes con un mismo diagnóstico, la inexistencia de pacientes con un único diagnóstico puro (la comorbilidad es la norma, no la excepción), etcétera, sin olvidar que distintos tipos de tratamientos pueden ser igualmente efectivos en un mismo cuadro clínico, sólo que para distintos perfiles de pacientes.

Las investigaciones clínicas, dotadas de una gran validez interna, permiten determinar el alcance terapéutico de un programa de intervención, es decir, su grado de eficacia. De este modo, se puede saber si los cambios observados en la variable dependiente son atribuibles al tratamiento y no a otras circunstancias (paso del tiempo, remisión espontánea, etcétera). Otra cosa es, sin embargo, la efectividad de dicho programa en la práctica clínica habitual, que es lo que le confiere la validez externa o ecológica. Un tratamiento deja de ser eficaz para convertirse en efectivo cuando se pueden generalizar los resultados obtenidos en la investigación -sometida a un riguroso control y en condiciones óptimas- a las situaciones clínicas reales (Becoña, 1999) (tabla 1).

TABLA 1

CRITERIOS DE ÉXITO EN LA TERAPIA

(Echeburúa y Corral, 2001)

\begin{tabular}{|c|c|c|}
\hline \multirow{2}{*}{$\begin{array}{l}\text { Validez } \\
\text { interna }\end{array}$} & & \multirow{2}{*}{ DEFINICIÓN } \\
\hline & $\begin{array}{c}\text { CONCEPTOS } \\
\text { FUNDAMENTALES }\end{array}$ & \\
\hline interna & Eficacia & $\begin{array}{l}\text { Resultados favorables } \\
\text { en un contexto de } \\
\text { investigación. }\end{array}$ \\
\hline & Efectividad & $\begin{array}{l}\text { Resultados favorables } \\
\text { en la práctica clínica } \\
\text { habitual. }\end{array}$ \\
\hline & Eficiencia & $\begin{array}{l}\text { Buenos resultados al } \\
\text { menor coste posible. }\end{array}$ \\
\hline
\end{tabular}

Sin embargo, en la investigación los pacientes son homogéneos entre sí (en cuanto a edad y sexo, por ejemplo) y puros, sin presentar 
comorbilidad con otros trastornos, y los terapeutas, que no suelen tener una gran experiencia clínica y que el paciente no puede elegir, están muy motivados. A su vez, los tratamientos, más bien cortos, se ajustan a una guía rígida y a un diario de sesiones. Por el contrario, en la práctica clínica habitual los pacientes están aquejados de varios trastornos simultáneamente, son heterogéneos y han sido sometidos, habitualmente, a tratamientos previos; los terapeutas cuentan con una experiencia clínica amplia y son objeto de elección por parte del paciente, al menos en la práctica privada; y, por último, los tratamientos son más largos y flexibles: no hay un número limitado de sesiones, el contenido del tratamiento es flexible y está sujeto a cambios, etcétera. En definitiva, no hay un isomorfismo entre la investigación y la clínica (tabla 2 ).

Tabla 2 Diferencias entre la investigación y la práctica clínica (Echeburúa y Corral, 2001)

\begin{tabular}{|c|c|c|}
\hline VARIABLES & INVESTIGACIÓN & PRÁCTICA CLÍNICA \\
\hline Pacientes & $\begin{array}{l}\text { - Homogéneos. } \\
\text { - Sin comorbilidad. } \\
\text { - Muy interesados por el tratamiento. }\end{array}$ & $\begin{array}{l}\text { - Heterogéneos. } \\
\text { - Con diversos trastornos. } \\
\text { - Menos interesados por la terapia. }\end{array}$ \\
\hline Terapeutas & $\begin{array}{l}\text { - Muy motivados, pero con poca } \\
\text { experiencia. }\end{array}$ & $\begin{array}{l}\text { - Con menor motivación, pero con } \\
\text { mayor experiencia profesional. }\end{array}$ \\
\hline Tratamiento & $\begin{array}{l}\text { - Contenido rígido. } \\
\text { - Número prefijado de sesiones. } \\
\text { - Menor número de sesiones. }\end{array}$ & $\begin{array}{l}\text { - Contenido flexible. } \\
\text { - Sin limitaciones prefijadas en el } \\
\text { número de sesiones. } \\
\text { - Mayor número de sesiones. }\end{array}$ \\
\hline Seguimiento & - Largos (6-12 meses). & - Cortos o inexistentes. \\
\hline
\end{tabular}

Por lo que se refiere al ámbito de la psicología clínica, las terapias cognitivo-conductuales y las terapias biológicas, por ejemplo, han experimentado un avance espectacular. En relación con estas últimas, se ha llegado incluso a calificar a los años 90 como la década del cerebro. Pero, sin embargo, no hay una correspondencia directa entre el descubrimiento de los receptores sinápticos y de los nuevos fármacos con la mejoría real de los pacientes con trastornos graves 
(esquizofrenia, autismo o trastorno bipolar, por ejemplo). Lo mismo ocurre en el ámbito de la psicopatología experimental. Tenemos hoy un conocimiento detallado de los procesos cognitivos implicados, por ejemplo, en los trastornos de ansiedad o en la depresión, pero no por ello ha habido un avance terapéutico claro del que puedan beneficiarse los pacientes. Esta última reflexión, sin llevar consigo una carga pesimista, trata de salir al paso de un cierto triunfalismo que se regodea en pseudoavances científicos sin repercusión clínica. En definitiva, no es un consuelo que la psiquiatría y la psicología clínica avancen a pasos agigantados, pero que los pacientes lo noten poco.

Por último, la organización de los Centros de Salud Mental, al nivel de la asistencia pública, puede constituir una rémora para la aplicación efectiva de las terapias empíricamente validadas. Si bien la primera visita se hace en un tiempo corto para no engrosar la lista de espera (y salvar de este modo las estadísticas oficiales), las siguientes visitas pueden tener un carácter mensual o bimestral por la presión asistencial existente. De este modo, el tratamiento psicológico responde a una infradosis terapéutica y se caracteriza más por un seguimiento que por un tratamiento propiamente dicho. No es por ello infrecuente dar el alta una vez remitida la fase aguda del trastorno y derivar al paciente al médico de atención primaria. De esta manera, se facilita la cronificación y el fenómeno de la puerta giratoria.

\section{Nuevas tendencias}

La terapia cognitivo-conductual (TCC) ha mostrado ser el desarrollo teórico más sólido disponible hasta la fecha y es la psicoterapia de referencia hoy por hoy. No ha habido progresos teóricos realmente significativos en las tres últimas décadas, pero se han acumulado estudios y datos fiables sobre su eficacia. Asimismo se han desarrollado las terapias en formatos grupales y se han aplicado a ellas también las nuevas tecnologías (programas de ordenador, realidad virtual, etcétera). Todo ello es positivo, siempre que no se desvirtúen los fundamentos mismos de la terapia cognitivo-conductual ni se minusvalore el énfasis necesario en la relación terapeuta-paciente. El 
lado negativo es el enfoque economicista aplicado a la terapia cognitivo-conductual, que puede llevar a frivolizar este enfoque. Así, por ejemplo, en Gran Bretaña se están formando especialistas en TCC, especialmente en universidades privadas, a un nivel de formación profesional (primary care mental health workers) sin la cualificación requerida, que tratan de aplicar mecánicamente diversas técnicas terapéuticas (relajación, control de contingencias, restructuración cognitiva, etcétera) y que constituyen una alternativa para atender a los pacientes con sintomatología no grave ansioso-depresiva que llegan a la Atención Primaria y que corren el peligro de colapsarla.

Hay nuevos desarrollos que pueden resultar de gran interés en ciertos contextos, como el crecimiento personal postraumático ( $\mathrm{Te}-$ deschi y Calhoun, 2004), basado en el aprendizaje a partir de los fracasos, o la entrevista motivacional (Miller y Rollnick, 1999), orientada a aquellos pacientes que no presentan una genuina motivación para el cambio (Pelechano, 2007).

Otros enfoques resultan más problemáticos. Así, por ejemplo, la terapia de aceptación y compromiso (Hayes, Follette y Linehan, 2004), considerada enfáticamente como la tercera ola en la terapia conductual (la primera fue el conductismo y la segunda el cognitivismo) no es propiamente una terapia psicológica. La ACT, que incorpora el yoga, la meditación zen, la relajación y la autohipnosis, es una terapia para alcanzar una vida más plena y cargada de sentido. Por ello, es una terapia que no se dirige a aliviar o eliminar los síntomas, sino a cosas más vagas (operativamente hablando), como recuperar una vida con sentido o aceptar la inevitabilidad del dolor o de los problemas.

\section{DEONTOLOGÍA PROFESIONAL}

Asimismo un reto de futuro es prestar una atención mayor a los aspectos éticos implicados en el quehacer profesional, especialmente a todo lo referido a la vulneración del derecho a la intimidad, a la mala praxis y a los informes periciales a petición de parte. De hecho, las reclamaciones más habituales de vulneración del Código Deontológico se relacionan con los problemas de las separaciones matrimoniales (informes psicológicos tendenciosos o de parte pre- 
sentados en el juicio como prueba), que se presentan en los Juzgados de Familia, o con la ruptura del secreto profesional (Del Río, 2005; Echeburúa, 2002).

\section{Práctica inadecuada}

Según el art. 4.7b de la LOPS, de aplicación para los psicólogos clínicos, la actuación del profesional sanitario estará basada en la evidencia científica y en los medios disponibles y amparados en guías y protocolos de práctica clínica y asistencial. Ello quiere decir, en un plano legal, que la evaluación de las terapias psicológicas permite establecer operativamente la práctica profesional inadecuada, que da lugar a hipotéticas responsabilidades civiles o penales.

En concreto, la mala praxis se configura cuando un profesional se separa de la lex artis (normas o guías de actuación profesional consensuadas) y actúa de forma técnicamente inadecuada. Esto es más claro cuando, como ocurre en la actualidad, comienzan a existir guías de actuación profesional en los distintos trastornos (Pérez, FernándezHermida, Fernández y Amigo, 2003).

Los psiquiatras han sido acusados por práctica inadecuada principalmente por causa de los diagnósticos erróneos, certificación de hospitalización impropia, suicidio del paciente, efectos dañinos de la terapia electroconvulsiva y de los psicofármacos (desarrollo de discinesia tardía con los neurolépticos), divulgación impropia de información y relaciones sexuales con los pacientes. Pero también se puede demandar a un psicólogo clínico por tratar sólo con psicoterapia un trastorno bipolar.

\section{CARACTERÍSTICAS PERSONALES DE LOS PSICÓLOGOS CLÍNICOS}

Por obvio que resulte decirlo, el psicólogo clínico va a estar en contacto con pacientes, es decir, con personas que sufren. Comprender al ser humano (o, en su caso, al paciente) es el requisito previo para cualquier actuación profesional.

Así, con independencia del ámbito concreto en el que se trabaje, los psicólogos clínicos deben aprender a mirar (no sólo ver) y a 
escuchar (no sólo oír), así como a empatizar, comprender y analizar. Ésta es la única manera de comunicarse adecuadamente con otras apersonas y de ayudar a resolver los problemas planteados.

Más allá de unas destrezas profesionales, se requieren en el psicólogo clínico unas características personales que faciliten el establecimiento de una alianza terapéutica con el paciente. Si esto es cierto en el ámbito de cualquier intervención profesional, lo es mucho más todavía en el campo específico de actuación de los psicólogos clínicos (la evaluación y los tratamientos psicológicos).

Por ello, no se puede desatender el peso específico de las características personales del psicólogo clínico en el resultado final de la terapia. Ante la constatación empírica de que hay una gran variabilidad en los logros obtenidos de unos terapeutas a otros, al margen de las técnicas terapéuticas utilizadas, el perfil personal idóneo del psicólogo clínico requiere estudios más precisos. Hay, sin embargo, algunas características básicas, ya conocidas, que facilitan la alianza terapéutica: equilibrio emocional, sentido común, capacidad de empatía, ausencia de rigidez y ganas genuinas de ayuda (Echeburúa y Corral, 2001).

Pero, además, los psicólogos deben contar con unas habilidades interpersonales que faciliten el trabajo en equipo o, al menos, el contacto con otros profesionales. Si los psicólogos clínicos están integrados en un Centro de Salud Mental o en un hospital, tienen que saber trabajar en equipo. Ello implica interactuar con otros profesionales de la salud (médicos de atención primaria, psiquiatras, enfermeras, etcétera), lo que obliga a saber utilizar un lenguaje común, a ser flexibles, a tener unas habilidades de comunicación, a marcarse objetivos concretos, a contar con un modelo integrador de salud, etcétera. Y si los psicólogos clínicos trabajan en la consulta privada, deben tener estas mismas habilidades para relacionarse con otros colegas, derivar casos a otros profesionales, saber relacionarse con los familiares de los pacientes, etcétera.

En resumen, no actúa necesariamente mejor con los pacientes el psicólogo clínico que más teoría sabe. Sin olvidar otras claves esenciales (conocimientos profesionales y disposición para estar al día, facilidad para adaptarse a los cambios, capacidad para integrarse en un equipo, motivación por la profesión), la inteligencia emocional, la 
autoestima apropiada y las habilidades interpersonales constituyen una herramienta fundamental para el desempeño adecuado de la psicología clínica.

\section{CONCLUSIONES}

La psicología clínica tiene que hacerse valer y estar atenta ante la respuesta que puede dar a las nuevas necesidades de una sociedad cambiante. Ello supone adaptarse a los tiempos que corren e implicarse en nuevas tareas, como la protocolización de actuaciones, la evaluación de resultados, los estudios de efectividad/eficiencia, la adopción de un lenguaje común con otros profesionales, etcétera.

Las investigaciones deben hacerse, fundamentalmente, en los Centros de Salud Mental o en los Servicios de Psiquiatría o Psicología Clínica porque los resultados obtenidos en estos ámbitos son más fácilmente generalizables que los conseguidos en universidades, en la práctica privada o con voluntarios. En este sentido, España o el Reino Unido, por ejemplo, pero no Estados Unidos, están en una situación óptima porque cuentan con una Sanidad Pública universal.

Al margen de las limitaciones de las investigaciones clínicas, éstas han supuesto ya un camino sin retorno en la práctica profesional de la psicología clínica. Por ello, las terapias psicológicas van a tender a estar estandarizadas, es decir, con una descripción precisa de los instrumentos de evaluación, el programa de tratamiento, el formato de aplicación (individual o grupal), el diario de sesiones, etcétera. Las guías de tratamiento efectivas se inscriben en el marco de terapias breves (10/15 sesiones) y específicas, lo que no es incompatible con la necesaria flexibilidad en la aplicación de estos tratamientos en función de las peculiaridades concretas de cada paciente.

Respecto al inmediato futuro, los enfoques terapéuticos basados en la resolución de problemas del aquí y ahora -un tema que puede ser común a distintas orientaciones- parecen desempeñar un papel importante en el desarrollo de los nuevos avances. De este modo, lo que puede explicar la eficacia similar de la terapia cognitiva y la terapia interpersonal en el tratamiento de la depresión es el acento puesto por una y otra en la estrategia de solución de problemas. Asimismo es sugerente la investigación reciente sobre el efecto potenciador de 
los tratamientos combinados (terapia cognitivo-conductual + psicofármacos) en diferentes cuadros clínicos. Los fármacos pueden actuar sobre la reducción de síntomas; los tratamientos psicológicos, sobre el aumento de competencias (Echeburúa y Corral, 2001).

No es exagerado afirmar, sin embargo, que las terapias no validadas empíricamente se utilizan con más frecuencia que los tratamientos basados en la evidencia y que, por tanto, hay un desfase entre lo que se sabe y lo que se hace. Por chocante que pueda resultar, lo que parece hoy efectivo dista de estar disponible para la mayoría de la población. De este modo, hacer que lo útil sea utilizado se convierte en una prioridad de actuación. La difusión no es algo que ocurre automáticamente, sino que está ligada a tres factores importantes: innovación (la aportación real de la nueva propuesta), canales de comunicación (el conocimiento de la nueva técnica por parte de los profesionales implicados) y transcurso del tiempo (necesario para romper con la resistencia al cambio en los profesionales) (Becoña, 1999)). De hecho, los clínicos se muestran muy reticentes a cambiar líneas de actuación y formas de abordar los problemas que tienen sobreaprendidas (Echeburúa y Corral, 2001).

Si bien la psicología clínica basada en la evidencia ha demostrado la superioridad de los enfoques cognitivo-conductuales sobre otro tipo de terapias, distan aún mucho de resolver problemas clínicos significativos: el incumplimiento de las prescripciones terapéuticas; la falta de motivación para el tratamiento en algunos trastornos (adicciones, parafilias, conductas violentas, etcétera); la terapia de los cuadros clínicos más graves de la psiquiatría pesada (psicosis, trastorno bipolar, etcétera). Y, además, la mejoría lograda en otros trastornos no siempre se corresponde con una calidad de vida similar a la de las personas normales. Todo ello es un reto para la investigación futura.

Por último, la psicología clínica basada en la evidencia es un camino irreversible. Pero todavía hay muchos cuadros clínicos sobre los que no hay tratamientos con una evidencia inequívoca. Por ello, la afirmación de que no hay pruebas sobre la eficacia de un determinado tratamiento no debe ser tomada como que existen pruebas de su ineficacia. 


\section{REFERENCIAS BIBLIOGRÁFICAS}

Avia, M.D. (Ed.) (2000). Cartas a un joven psicólogo. Madrid: Alianza. Bados, A., García, E. \& Fusté, A. (2002). Eficacia y utilidad clínica de la terapia psicológica. Revista Internacional de Psicología Clínica y de la Salud, 2, 477-502.

Bayés, R. (1984). ¿Por qué funcionan las terapias comportamentales?. Anuario de Psicología, 30-31, 127-147.

Becoña, E. (1999). La discrepancia entre la investigación y la práctica clínica de la terapia de conducta. Revista de Psicopatología y Psicología Clínica, 4, 71-103.

Del Río, C. (2005). Guía de ética profesional en psicología clínica. Madrid: Pirámide.

Echeburúa, E. (1998). ¿Qué terapias psicológicas son eficaces? Un reto ante el 2000. Revista de Psicopatología y Psicología Clínica, 3, 149-160.

Echeburúa, E. (2002). El secreto profesional en la práctica de la psicología clínica y forense: alcance y límites de la confidencialidad. Análisis y Modificación de Conducta, 28, 498-504.

Echeburúa, E. \& Corral, P. (2001). Eficacia de las terapias psicológicas: de la investigación a la práctica clínica. Revista Internacional de Psicología Clínica y de la Salud, 1, 181-204.

Echeburúa, E., Corral, P. \& Salaberría, K. (2005). Reflexiones ante la formación de los psicólogos. Retos de futuro. Análisis y Modificación de Conducta, 31, 175-188.

Hayes, S.C., Follette, V.M. \& Linehan, M.M. (2004). Mindfulness and acceptance: expanding the cognitive behavioural tradition. New York: Guilford Press.

Miller, W.R. \& Rollnick, S. (1999). La entrevista motivacional. Preparar para el cambio de conductas adictivas. Barcelona: Paidós.

Pelechano, V. (2007). Viejas y nuevas cuestiones en las viejas y nuevas terapias psicológicas. Revista de Psicopatología y Psicología Clínica, 12, 71-90.

Pérez, M., Fernández-Hermida, J.R., Fernández, C. \& Amigo, I. (Eds.) (2003). Guía de tratamientos psicológicos eficaces. Madrid: Pirámide. 3 vols. 
Tedeschi, R.G. \& Calhoun, L.G. (2004). A clinical approach to posttraumatic growth. En P.A. Linley \& S. Joseph (Eds.). Positive psychology in practice. Hoboken, NJ: John Wiley \& Sons.

Turner, S.M., Beidel, D.C., Spaulding, S.A. \& Brown, J.M. (1995). The practice of behavior therapy: A national survey of cost and methods. The Behavior Therapist, 18, 1-4. 
